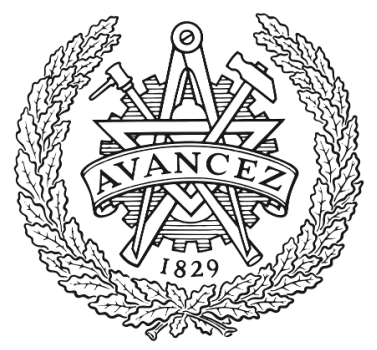

CHALMERS

UNIVERSITY OF TECHNOLOGY

\title{
Teachers' Views on the Use of Empathic Robotic Tutors in the Classroom
}

Downloaded from: https://research.chalmers.se, 2023-04-26 12:09 UTC

Citation for the original published paper (version of record):

Serholt, S., Barendregt, W., Leite, I. et al (2014). Teachers' Views on the Use of Empathic Robotic Tutors in the Classroom. The 23rd IEEE International Symposium on Robot and Human Interactive Communication: 955-960. http://dx.doi.org/10.1109/ROMAN.2014.6926376

N.B. When citing this work, cite the original published paper. 


\title{
Teachers' Views on the Use of Empathic Robotic Tutors in the Classroom*
}

\author{
Sofia Serholt, Wolmet Barendregt, Iolanda Leite, Helen Hastie, Aidan Jones, Ana Paiva, Asimina \\ Vasalou and Ginevra Castellano
}

\begin{abstract}
In this paper, we describe the results of an interview study conducted across several European countries on teachers' views on the use of empathic robotic tutors in the classroom. The main goals of the study were to elicit teachers' thoughts on the integration of the robotic tutors in the daily school practice, understanding the main roles that these robots could play and gather teachers' main concerns about this type of technology. Teachers' concerns were much related to the fairness of access to the technology, robustness of the robot in students' hands and disruption of other classroom activities. They saw a role for the tutor in acting as an engaging tool for all, preferably in groups, and gathering information about students' learning progress without taking over the teachers' responsibility for the actual assessment. The implications of these results are discussed in relation to teacher acceptance of ubiquitous technologies in general and robots in particular.
\end{abstract}

\section{INTRODUCTION}

Intelligent tutoring systems (ITS) have long explored the benefits of personalized education. One area of research has focused on developing lifelike virtual characters or animated pedagogical agents (APA) for ITS, offering the possibility of engaging and motivating students within a learning task through verbal and nonverbal communication $[1,2]$. Previous research has demonstrated that a virtual agent's level of embodiment plays a significant role. Indeed, several studies suggest that people's perception of robots is qualitatively different from their perception of virtual agents and is attributable to robots' physical embodiment [3]. Experiments comparing robots with virtual representations have repeatedly shown that the robotic embodiment is preferred by end users in terms of social presence $[4,5]$, enjoyment and performance [6], and also led to increased learning gains [7].

Therefore, it seems that robots can enrich traditional ITS because of the sense of presence they provoke [8] and the

*Research partially supported by the European Commission (EC) and funded by the EU FP7 ICT-317923 project EMOTE.

Sofia Serholt and Wolmet Barendregt are with the Department of Applied Information Technology, University of Gothenburg, Gothenburg, Sweden (phone: +46-737-108076; e-mail: [sofia.serholt, wolmet.barendregt]@ ait.gu.se).

Iolanda Leite and Ana Paiva were with GAIPS, INESC-ID and Instituto Superior Tecnico, Lisboa, Portugal. Leite is now with the Social Robotics Lab, Yale University, New Haven, CT 06520 USA (e-mail: iolanda.leite@yale.edu, ana.paiva@inesc-id.pt).

Helen Hastie is with the School of Mathematical and Computer Science, Heriot-Watt University, Edinburgh, UK (e-mail: h.hastie@hw.ac.uk).

Aidan Jones and Ginevra Castellano are with the School of Electronic, Electrical and Computer Engineering, University of Birmingham, Edgbaston, Birmingham, B15 2TT, UK (e-mail: [axj100, g.castellano]@bham.ac.uk).

Asimina Vasalou is with the London Knowledge Lab, Institute of Education, London, UK (e-mail: minav@luminainteractive.com). potential value of introducing educational service robots in the classroom has led to a rapidly expanding field [9-18]. Unlike hands-on experimental robotics used within technical school subjects, educational service robots take "anthropomorphized forms to substitute or support teachers" [19]. These robots may act as peer learning companions, tutors or mentors [20].

Inspired by the possible applications of Human-Robot Interaction (HRI), and the promising results about embodiment, our project is developing an intelligent robotic tutor with perceptive and empathic capabilities that will deliver geography and sustainability education to late primary school students between the ages of 11-13 [21]. The aim is to design a fully autonomous robot using a Nao torso, developed by Aldebaran Robotics. Previous research endeavors exploring the impact of educational service robots within authentic educational settings have mainly been used for learning language, science and technology. To our knowledge, the subjects of geography and sustainable development have not yet been explored.

The motivation for equipping the robot with perceptive and empathic capabilities has partly emerged from the growing interests surrounding affect recognition within the field of ITS, able to detect, interpret and respond to users' emotional and affective states [22, 23]. When it comes to human-human tutoring situations, it is of utmost importance that the teacher is sensitive to the affective state of the student, since this will allow for adaptations in the pedagogical strategy employed by the teacher. For example, if a teacher senses that a student is losing interest in an assignment and as a consequence displays boredom, the pedagogically appropriate response is for the teacher to adjust his or her teaching strategy accordingly. This ability is considered to be one of the strengths of human tutoring, and is thus an important trait before agents can interact with users in a socially acceptable way [24]

A key learning aim in geography is to teach students spatial navigation, which is achieved through the navigation of maps and the use of symbols to give one example. We envision the robot taking the role of a tutor while students interact with age appropriate maps on a touchscreen table. Three design sensibilities ground our project: first, we want to endow the robot with sound educational strategies approximating those that teachers use to motivate and stimulate their students; second, we aspire to build a robot with perceptive capabilities that recognizes students' engagement - or disengagement - and delivers its teaching strategies empathically; third, the robotic should seamlessly integrate into a classroom setting. 
This paper details design research conducted to unpack our third design sensibility. We note that previous design research concerning social robots or educational service robots has tended to focus on the primary users, i.e. the students $[14,25,26]$. Surprisingly, teachers' views on the use of such robots have not yet received as much attention until rather recently [27]. Such explicit attention to the perspectives of the potential adopters is important for any technology implementation [28, 29], and particularly for robots in education. For example, a recent survey of European attitudes towards robots in society [30] revealed that only $3 \%$ of the population believed that robots should be used for educational purposes, and $34 \%$ believed that educational robots should be banned completely. This exemplifies the importance of collaborating with a wide range of stakeholders, so that their concerns may be taken into account.

Our goal in involving teachers is two-fold. First, if robotic tutors are to be introduced in schools, there is a need to establish whether teachers - who act as the gatekeepers for any new technologies - will accept and use them, without which the impact of this new technology is likely to decline. Second, given their years of experience, teachers have rich knowledge around potential difficulties and pragmatic issues that may arise within school settings and create barriers to the adoption of robots. To answer these open questions with a view to fold them back to design, we involved teachers during the early design deliberations of our robotic tutor. In the next section, we describe the procedure of the initial set of explorative interviews.

\section{METHOD}

We recruited teachers in local public and private schools who agreed to participate. The teachers at these particular schools were expected to provide us with continuous feedback throughout the duration of the project. In total, eight teachers ( 5 female) from four different countries (England, Scotland, Portugal, Sweden) between the ages of 25 and 48 $(\mathrm{M}=42, \mathrm{SD}=10)$ participated in the interviews. Our youngest participant had been a teacher for only one year, while our oldest participant had 26 years of experience.

The interviews were conducted in the local schools in which teachers worked and lasted, on average, 30 minutes each. All interviewers followed the same procedure in the four countries: they briefly introduced the goals of the project and one plausible scenario for a robotic tutor (the robot aiding a student in a map-reading task). After that, a set of open-ended and semi-structured questions were presented. These included questions on the plausibility of having a robotic tutor in their classes, the ideal number of students interacting with the robot at the same time, the different roles that the robot could play, in which ways the robot could monitor students, and the main pros and cons about having such technology in classrooms (e.g., when would they think a robotic tutor hindered or aided their work). There were no initial hypotheses; rather the aim was to explore the general idea of integrating robotic tutors within educational settings from teachers' points of view. As such, the flexibility of having open-ended questions allowed for teachers to steer the discussions in different directions.
Most interviews were audio recorded except in two cases where, due to delays in audio permission requests, extensive notes were taken (also containing relevant quotes from the teachers). All recorded interviews were transcribed and if necessary translated to English. Thereafter, five researchers read through all transcriptions to detect common themes, which they noted as comments in the transcripts. Since the interviews constituted an initial exploration of the potential concerns or design issues anticipated by teachers with regard to their practical experience, the aim was not to conduct a thorough analysis of these particular interview situations. As such, no coding scheme was devised for analysis. Below, the recurring themes as agreed upon by the researchers through iterative sessions are presented.

\section{RESULTS}

The main themes resulting from the performed qualitative analysis are presented in individual sections below. First, the aspects relating to practical concerns about potential disruptions of the technology are presented. Second are the practical benefits that teachers envision from using the technology. Third are how robots may contribute to the learning situation for students; and fourth, how teachers viewed the perceptive and empathic capabilities. To keep their identities anonymous, teachers are labeled within the range of 1 to 8 , where T1, T2 and T3 are from Portugal, T4 is from England, T5 to T7 are from Sweden and T8 is from Scotland.

\section{A. Robots as a Disruptive Technology}

Teachers expressed general concerns that the presence of robotic tutors might introduce competition between students who would want to interact with the robot. This was predicted to result in increased administrative and "conflict resolution" workload for the teacher who would have to keep track of each student's time spent working with the robot, which can be a practical disadvantage due to the time constraints they face. In the words of one teacher:

Problems would arise if there aren't enough robots and not enough students could use them... if it becomes a competitive situation, and you have to keep track of, for example, that Pelle has been using the robot this week, and now he can't use it until week 7 again. Then there'd be a lot of administration around it. The fairness aspect is very important for kids (T5).

However, another teacher pointed out that competition would probably decrease in step with the novelty effect wearing off. As she argued,

When it becomes an everyday occurrence, the novelty effect will vanish, and the students will lose sight of the new, and feel more... Then I think it'll be ok. When everyone has gotten to try it once (T7).

Another teacher even noted that the wearing-off effect could eventually result in a loss of interest:

People would love to use it and get something out of it. The concern is that they love a new gadget, but that it loses its novelty quickly (T8). 


\section{B. Robots Designed For Classrooms}

Teachers envisioned the robot working in the context of existing classroom practices. In particular, they suggested constructing a class activity with the robot conveying educational material to groups of students, for example through station rotation:

So I could be working with another group of students on another activity, maybe a reading or writing activity, or something like that, whilst another group was working on, say, the map exercise, but with that robotic assistant to be there to help them (T3).

Teachers expressed the importance of developing a robot that would manage group work, since strictly having individual sessions could become extremely difficult to practically manage within one 40-minute lesson of geography per week:

Well, that depends on the access to robots, because I wouldn't... If there would be only one robot and only one student could work with it, then... That is not enough (T5).

Some teachers additionally discussed the issues that often arise during group work and could occur when students were left alone with the robot:

Also, if there is a group of students using it, or being helped by the robot, can it adapt to each student? I'm thinking they can be on quite different levels for example. Or where one student can take over and be very controlling if it's a game for example, and be very dominant. In those cases it's possible for me as a teacher to intervene and even this out. Is that something that the robot will be able to do? Because that always happens during group work, they are different, they take different roles, and then to be able to make sure that it is maximized learning for each participant, so that not only some benefit (T6).

But someone would have to supervise the students interacting with the robot. Leaving them alone at this age, it's a bit unpredictable (T1).

\section{Robots supporting teachers}

Teachers recognized that if robots can operate autonomously they could be beneficial for their teaching practice by reducing their workload both in terms of teaching and administrative tasks. In other words, if robots could function without much interference from the teacher, teachers believed that robotic tutors could provide a solution to the time constraints they face on a daily basis.

As I said before about feeling insufficient, when you don't have the time. If a robot could be there to motivate, maybe push, aid and help, as well as deepen certain concepts, to push the students to think an extra step, and not just take the easy road (T7).

That I feel that I don't need to supervise the students to the same extent as a teacher, but that I know that it... Even if it's not a person, there is someone there who can help in some way. So it would be like an extra assistant, you could say (T6).
Moreover, teachers pointed out that they wanted the robot to contain a sort of automated assessment database, where they could check each student's progress; something which is also required for the personalization of any learning technology. However, it was made clear that they did not want to grant the application freedom to actually grade their students, stressing the importance of the teacher being in control. In addition, students would need to be aware of exactly what is documented about them in order to preserve their integrity.

I think it would benefit the teacher to find out what precisely they have been working on. What core content are they working with? Umm... and maybe what learning objectives you can expect to see them developing... Not that you have the robot provide an assessment and a grade such as E, C or A... Rather which skills you should expect to see, and if you can test that some other way (T5).

So long as everybody's clear about the parameters, and everybody's clear about what's being recorded, and what information is being stored, because that's an issue obviously (T3).

A lot of the assessment takes place during the interaction with the teacher, I don't think there are a lot of teachers who would appreciate it if, "Ok, so you can go and tested by him, and he'll tell me what grade you got, and I will mark it down... (T5)

\section{Aspirations for Teaching and Learning}

Most teachers conceived of robotic tutors as peers (in relation to students) that can guide and motivate students through several learning tasks. One teacher also highlighted the fact that robots in educational settings can promote independent leaning:

It enables more independent learning on the part of the student, but supported independent learning (T4).

As teachers valued providing personalized responses to students, they thought the robot could become a resource if able to encourage students to think an extra step when they would not have the time to do so:

Because it's very easy for students to just, "Well, I'm done, so I'm finished!" But to have someone say, "Well, how about..? Can you think...?" That's always what you are trying to do as a teacher, but a robot might as well say, "Yes, but can you extend that? Can you extend the text a bit? Can you think about what consequences this will result in?" Those are the things that you would like to do, but don't have the time to when the class is working (T6).

\section{E. Forming Social and Affective Bonds with Robots}

Similar to other ubiquitous technologies, when discussing humanoid robots aimed for educational settings, fears are induced regarding the risk of robots replacing human-human interaction. We noticed these concerns in some of the teachers we interviewed. At first, they seemed inclined to carefully assess whether or not we were working on robots intended to replace them. When researchers informed them that this was not case, they went on to question if a social 
connection was even possible, partly due to lack of faith in or knowledge about the recent technology advances:

Then it's the part about feelings... I can feel a bit ambivalent... Can a robot, or I don't know if it can completely replace a human being when it comes to emotions (T6).

To me it seems utopian to analyze behaviors if it does not know whether the kid is focused or not (T2).

And that's where the human element, I think, personally, I can't see how that could ever be replaced (T3).

\section{CONCLUDING DiscuSSION}

Our interviews with teachers revealed a set of implications and concerns for designing and evaluating robotic tutors for use in classrooms.

A first design concern is that robotic tutors should avoid creating administrative overhead for the teacher. In line with this, teachers emphasized that access to the robot should fit with existing classroom practices and social norms. Robots should be used fairly independently by individuals, or preferably in groups so that access is distributed. Deploying a robot in a group setting, requires monitoring and understanding of social interactions, and inevitably introduces new technical challenges. Secondly, robots should support the teacher's role responsibly. A number of teachers proposed that robots assist in recording information that is later used for assessment. At the same time however, they were clear that grading responsibility must remain with the teacher. While this principle is in accordance with open learner models, which are tools that support teachers [31], it goes beyond many learning analytics visualizations (e.g. dashboards [32]), given that teachers emphasized the importance of accessing understanding and competencies.

Alongside these design implications, we also gained insight into teachers' attitudes and expectations of robotic technologies. Building on the hopes and hypes of technologyenhanced learning more generally, teachers believed that robots could potentially introduce more independent forms of learning and personalized tutoring [33]. However, in reference to the affective capability of our robot, teachers were unable to sufficiently grasp how the technology would work toward reflecting on the potential social and moral implications identified by researchers in the area of HRI [34].

Altogether, these results point out the need for longitudinal evaluations which take into account the whole classroom and learning situation, not just the immediate learning effects. Yet, this requires that researchers understand the measures deemed important by the key stakeholders. Scanlon and Isroff [35] emphasize the importance of developing a rich set of criteria before evaluating whether technological solutions within educational institutions are feasible. These criteria should, in turn, be based on a holistic view of the educational setting informed by the participants within that setting. Hence, teachers' involvement throughout the design process is essential to understand the classroom situation in which the robotic tutors will be integrated.

Yet, this will require that the involved teachers also truly understand what this new technology can do, and how it may come to affect their practice. As argued above, affect recognition and empathic technologies were difficult concepts to grasp. Participating teachers questioned whether it is even possible to develop such technologies, and consequently, tended to identify issues that had a clear connection to their daily practice. While our interviews make a contribution by providing insight into the importance of adapting new technologies to the pragmatic circumstances that teachers and students are faced with, they do not provide an understanding of teachers' moral and ethical perspectives on their students prospectively developing social connections with a robot. Gathering insights in this area before an actual implementation is made is crucial, so that it is clear what traits and behaviors in an empathic tutor are deemed acceptable by the potential adopters of the technology.

\section{FUTURE WORK}

Two aspects that received relatively limited attention were attitudes and concerns about how the presence of such a robotic tutor could potentially change the social dynamics of the classroom situation, and the aforementioned empathic connection between robots and students. We will therefore continue our user-centered design activities with the teachers to address these aspects.

First, we would like to delve deeper into teachers' views on how the presence of the robotic tutors could change the classroom situation. Imagining a potential impact of an unfamiliar technology is no easy task. As such, researchers cannot rely on respondents to have genuine understandings of the implications inherent with novel technologies. At the same time, eliciting perceptions and attitudes of potential users is vital in order for the particular technology to have a future impact in line with the expectations of the users. These issues relate to the Collingridge dilemma, i.e. that "at early development stages consequences are difficult to predict whereas at later stages where consequences become clearer the trajectory of the development becomes more difficult to change" [36].

Bearing in mind the difficulties with eliciting people's true perceptions and attitudes, we aim to provide teachers with videos or scenarios inspired by the ContraVision approach. This approach was described by Mancini et al. [37] for investigating users' reactions to a fictional dieting support system. Unlike other studies exploring perceptions of ubiquitous technologies, where participants may be provided with a single version of a fictive story vignette, in the ContraVision approach, two representations of the same technology, one positive and one negative, are used to elicit a wide spectrum of the perceptions that people may have of the future technology.

Second, in order to elicit teachers' responses on the actual behavior of the robots towards students, we are planning to have teachers acting as wizards in a Wizard-of-Oz (WoZ) study, which is a rather novel approach. Usually, WoZ interfaces are controlled by their developers or other researchers involved in the project; yet these usually lack the pedagogical experience necessary to anticipate students' potential difficulties or be able to adapt to students in a pedagogically appropriate way. By involving teachers closely during all stages of development, we expect not only to 
design more useful and engaging robotic tutors for students modeled by the timely responses demonstrated by human teachers but, more importantly, develop valuable educational tools that teachers actually want to have in their classroom.

\section{ACKNOWLEDGMENT}

We would like to thank the teachers at Leteboskolan, Horred, Sweden; Haybridge High School, Birmingham, UK; and Agrupamento de Escolas S. Julião da Barra, Portugal. This work was partially supported by the European Commission (EC) and was funded by the EU FP7 ICT317923 project EMOTE (www.emote-project.eu). The authors are solely responsible for the content of this publication. It does not represent the opinion of the EC, and the EC is not responsible for any use that might be made of data appearing therein.

\section{REFERENCES}

[1] W. L. Johnson, J. W. Rickel, and J. C. Lester, “Animated Pedagogical Agents: Face-to-Face Interaction in Interactive Learning Environments," International Journal of Artificial Intelligence in Education, vol. 11, pp. 47-78, 2000.

[2] S. W. McQuiggan, J. P. Rowe, and J. C. Lester, "The effects of empathetic virtual characters on presence in narrative-centered learning environments," in Proceedings of the SIGCHI Conference on Human Factors in Computing Systems, Florence, Italy, 2008, pp. 1511-1520.

[3] K. M. Lee, Y. Jung, J. Kim, and S. R. Kim, "Are physically embodied social agents better than disembodied social agents?: The effects of physical embodiment, tactile interaction, and people's loneliness in human-robot interaction," International Journal of Human-Computer Studies, vol. 64, no. 10, pp. 962-973, 2006.

[4] C. Kidd, "Sociable Robots: The Role of Presence and Task in HumanRobot Interaction," Georgia Institute of Technology, Massachusetts Institute of Technology, 2003.

[5] W. A. Bainbridge, J. W. Hart, E. S. Kim, and B. Scassellati, "The Benefits of Interactions with Physically Present Robots over VideoDisplayed Agents," International Journal of Social Robotics, vol. 3, no. 1, pp. 41-52, 2011.

[6] C. Bartneck, "eMuu - An embodied emotional character for the ambient intelligent home," Industrial Design, 2002.

[7] D. Leyzberg, S. Spaulding, M. Toneva, and B. Scassellati, "The Physical Presence of a Robot Tutor Increases Cognitive Learning Gains," in Proceedings of the 34th Annual Conference of the Cognitive Science Society, 2012.

[8] C. D. Kidd, and C. Breazeal, "Effect of a robot on user perceptions," in Intelligent Robots and Systems, 2004. (IROS 2004). Proceedings. 2004 IEEE/RSJ International Conference on, 2004, pp. 3559-3564 vol.4.

[9] C.-W. Chang, J.-H. Lee, P.-Y. Chao, C.-Y. Wang, and G.-D. Chen, "Exploring the Possibility of Using Humanoid Robots as Instructional Tools for Teaching a Second Language in Primary School.," Educational Technology \& Society, vol. 13, no. 2, pp. 13-24, 2010.

[10] S. Eimler, A. von der Pütten, U. Schächtle, L. Carstens, and N. Krämer, "Following the White Rabbit - A Robot Rabbit as Vocabulary Trainer for Beginners of English," USAB 2010, LNCS 6389, G. Leitner, M. Hitz and A. Holzinger, eds., Berlin Heidelberg: Springer-Verlag, 2010.

[11] T. Fumihide, and S. Matsuzoe, "Children Teach a Care-Receiving Robot to Promote Their Learning: Field Experiments in a Classroom for Vocabulary Learning," Journal of Human-Robot Interaction, vol. 1, no. 1, pp. 78-95, 2012.

[12] J. Janssen, C. Wal, M. Neerincx, and R. Looije, "Motivating Children to Learn Arithmetic with an Adaptive Robot Game," Social Robotics, Lecture Notes in Computer Science B. Mutlu, C. Bartneck, J. Ham, V. Evers and T. Kanda, eds., pp. 153-162: Springer Berlin Heidelberg, 2011.
[13] T. Kanda, T. Hirano, D. Eaton, and H. Ishiguro, "Interactive Robots as Social Partners and Peer Tutors for Children: A Field Trial," HumanComputer Interaction, vol. 19, pp. 61-84, 2004.

[14] H. Kozima, and C. Nakagawa, "Longitudinal Child-Robot Interaction at Preschool," in AAAI Spring Symposium: Multidisciplinary Collaboration for Socially Assistive Robotics, 2007, pp. 27-32.

[15] E. Lee, and Y. Lee, "A Pilot Study of Intelligent Robot Aided Education," in ICCE Conference on Advanced Learning Technologies, Open Contents, \& Standards, 2008.

[16] H. Ryu, S. S. Kwak, and M. Kim, "A Study on External Form Design Factors for Robots as Elementary School Teaching Assistants," in 16th IEEE International Conference on Robot \& Human Interactive Communication, Jeju, Korea, 2007, pp. 1046-1051

[17] M. Saerbeck, T. Schut, C. Bartneck, and M. D. Janse, "Expressive Robots in Education - Varying the Degree of Social Supportive Behavior of a Robotic Tutor," in CHI 2010, Atlanta, Georgia, USA, 2010, pp. 1613-1622.

[18] Z. You, C. Shen, C. Chang, B. Liu, and G. Chen, "A Robot as a Teaching Assistant in an English Class," in Proceedings of the Sixth International Conference on Advanced Learning Technologies, 2006.

[19] J. Han, "Robot-Aided Learning and r-Learning Services," HumanRobot Interaction, D. Chugo, ed., InTech, 2010, p. 288.

[20] O. Mubin, C. J. Stevens, S. Shahid, A. A. Mahmud, and J. Dong, "A review of the applicability of robots in education," Technology for Education and Learning, pp. 1-7, 2013.

[21] S. Serholt, and W. Barendregt, "The EMOTE project: Teaching geography through embodied-perceptive tutors for empathic-based learning in a game environment," in European Conference on Games Based Learning, Portugal, 2013.

[22] S. T. V. Alexander, "An affect-sensitive intelligent tutoring system with an animated pedagogical agent that adapts to student emotion like a human tutor," Computer Science, Massey University, Albany, New Zealand, 2007

[23] W. Burleson, "Affective Learning Companions: Strategies for Empathetic Agents with Real-Time Multimodal Affective Sensing to Foster Meta-Cognitive and Meta-Affective Approaches to Learning, Motivation, and Perseverance," Massachusetts Institute of Technology, 2006.

[24] G. Castellano, I. Leite, A. Pereira, C. Martinho, A. Paiva, and P. W. McOwan, "Affect Recognition for Interactive Companions: Challenges and design in real world scenarios," Journal on Multimodal User Interfaces, vol. 3, no. 1-2, pp. 89-98, 2010.

[25] E. Hyun, and S. Son, "Is Robot Alive? Young Children's Perception of a Teacher Assistant Robot in a Classroom," Korean Journal of Child Studies, vol. 32, no. 4, pp. 1-14, 2011.

[26] T. Kanda, M. Shimada, and S. Koizumi, "Children learning with a social robot," in Proceedings of the seventh annual ACM/IEEE international conference on Human-Robot Interaction, Boston, Massachusetts, USA, 2012, pp. 351-358.

[27] M. Fridin, and M. Belokopytov, "Acceptance of socially assistive humanoid robot by preschool and elementary school teachers," Computers in Human Behavior, vol. 33, pp. 23-31, 4//, 2014.

[28] P. Mishra, and M. J. Koehler, "Technological Pedagogical Content Knowledge: A Framework for Teacher Knowledge," Teachers College Record, vol. 108, no. 6, pp. 1017-1054, 2006.

[29] D. L. Russell, and A. Schneiderheinze, "Understanding Innovation in Education Using Activity Theory," Educational Technology \& Society, vol. 8, no. 1, pp. 38-53, 2005.

[30] European Commission, Public Attitudes Towards Robots, http://ec.europa.eu/health/eurobarometers/index en.htm, 2012.

[31] P. Reimann, S. Bull, and P. Ganesan, "Supporting the Development of 21st Century Skills: Student Facilitation of Meetings and Data for Teachers," in Proc. Workshop Towards Theory and Practice of Teaching Analytics, 2012.

[32] K. Verbert, E. Duval, J. Klerkx, S. Govaerts, and J. Santos, "Learning Analytics Dashboard Applications," American Behavioral Scientist, vol. 57, no. 10, pp. 1500-1509, 2013.

[33] N. Selwyn, Education and Technology: Key Issues and Debates, London: Continuum, 2011.

[34] P. H. Kahn, H. E. Gary, and S. Shen, "Children's Social Relationships With Current and Near-Future Robots," Child Development Perspectives, vol. 7, no. 1, pp. 32-37, 2013. 
[35] E. Scanlon, and K. Isroff, "Activity Theory and Higher Education: evaluating learning technologies," Journal of Computer Assisted Learning, vol. 21, pp. 430-439, 2004.

[36] B. C. Stahl, N. McBride, K. Wakunuma, and C. Flick, "The empathic care robot: A prototype of responsible research and innovation," Technological Forecasting \& Social Change, pp. 1-12, 2013.

[37] C. Mancini, Y. Rogers, A. K. Bandara, T. Coe, L. Jedrzejczyk, A. N. Joinson, B. A. Price, K. Thomas, and B. Nuseibeh, "Contravision: exploring users' reactions to futuristic technology," in Proceedings of the SIGCHI Conference on Human Factors in Computing Systems, Atlanta, Georgia, USA, 2010, pp. 153-162. 